\title{
Downregulation of secreted protein acidic and rich in cysteine in human trabecular meshwork cells
}

\author{
YANG FU $^{1,2^{*}}$, LIYING LUO ${ }^{1,2^{*}}$, YING FAN $^{1,2}$ and MIN TANG ${ }^{1,2}$ \\ ${ }^{1}$ Department of Ophthalmology, Shanghai General Hospital, Shanghai Jiao Tong University, \\ School of Medicine; ${ }^{2}$ Shanghai Key Laboratory of Fundus Disease, Shanghai General Hospital, Shanghai 200080, P.R. China
}

Received July 27, 2020; Accepted June 30, 2021

DOI: $10.3892 /$ etm.2021.10560

\begin{abstract}
Secreted protein acidic and rich in cysteine (SPARC) may play a notable role in aqueous humor outflow through the trabecular meshwork (TM). SPARC is a potential therapeutic target in glaucoma, and the mechanism by which it regulates intraocular pressure remains unclear. The present study aimed to observe the effects of SPARC in human TM cells (HTMCs) in vitro. SPARC was downregulated by recombinant lentiviral vectors in HTMCs, and the subsequent levels of F-actin expression, zonula occludens-1 (ZO-1) expression and cellular phagocytosis were observed and calculated. It was revealed that after $48 \mathrm{~h}$ of culture, the expression levels of SPARC, F-actin and ZO-1 were significantly decreased in the lentivirus group compared with those in the blank control and empty vector control groups. The downregulation of SPARC promoted phagocytosis in HTMCs after 24 or $48 \mathrm{~h}$ of culture. This indicated that the downregulation of SPARC decreased the expression levels of the cytoskeleton-associated proteins F-actin and ZO-1, promoted phagocytosis in HTMCs and may affect the outflow of aqueous humor via the TM pathway.
\end{abstract}

Correspondence to: Dr Min Tang, Department of Ophthalmology, Shanghai General Hospital, Shanghai Jiao Tong University, School of Medicine, 100 Haining Road, Hongkou, Shanghai 200080, P.R. China E-mail: tmsmile@sina.com.cn

*Contributed equally

Abbreviations: POAG, primary open angle glaucoma; IOP, intraocular pressure; TM, trabecular meshwork; HTMC, human trabecular meshwork cell; ECM, extracellular matrix; JCT, juxtacanalicular tissue; SPARC, secreted protein acidic and rich in cysteine; ZO-1, zonula occludens-1; DMEM, Dulbecco's modified Eagle's medium; FBS, fetal bovine serum; MYOC, myocilin; RT-qPCR, reverse transcription-quantitative polymerase chain reaction; PBS, phosphate-buffered saline

Key words: matricellular protein, secreted protein acidic and rich in cysteine, F-actin, zonula occludens-1, trabecular meshwork

\section{Introduction}

Glaucoma is a leading cause of irreversible blindness worldwide, and its prevalence increases with age. In 2013, the global prevalence of glaucoma for people aged 40-80 years was $3.54 \%$, and the number of people in this age range with glaucoma worldwide was estimated to be 64.3 million, increasing to 76.0 million in 2020 and 111.8 million in 2040 (1). Intraocular pressure (IOP) is a notable modifiable risk factor that depends on the balance of aqueous humor production and outflow, and it is thought that prolonged IOP elevation leads to optic nerve damage. However, the existing treatments to control IOP, including anti-glaucoma medication, laser therapy and surgery, cannot meet clinical needs. In 2010, 2.1 million people went blind and 4.2 million people were visually impaired due to glaucoma around the world (2).

Secreted protein acidic and rich in cysteine (SPARC), also known as osteonectin or BM-40, is a promising matricellular protein that is involved in glaucoma and functions primarily to promote extracellular matrix (ECM) deposition (3). In human eyes, it is distributed throughout the trabecular meshwork (TM), with pronounced expression in the juxtacanalicular tissue (JCT) (3). Research indicates that, in porcine TM cells, SPARC is one of the most highly upregulated genes following mechanical stretching (4), while IOP drops by $15-20 \%$ in SPARC-null mice (5). In addition, elevated SPARC expression has been found in the iris of patients with primary open angle glaucoma (POAG) (6). Together, these indicate that SPARC could be involved in POAG, potentially by compromising IOP regulation. SPARC is a potential therapeutic target, and the mechanism by which it regulates IOP remains to be clarified.

Physiologically, the TM mediates major aqueous humor outflow and maintains the IOP. Previous research indicates that the remodeling of the TM cellular cytoskeleton and ECM metabolism has significant effects on the regulation of IOP within TM tissues $(7,8)$. The present study hypothesized that SPARC could regulate the expression of cytoskeleton-associated proteins and cytoskeletal rearrangement in TM cells, and in turn affect cell-ECM interactions, just as it does in some other tissues $(9,10)$. To test this hypothesis, the regulation of SPARC was observed with regard to F-actin, zonula occludens-1 (ZO-1) and phagocytosis in human TM cells in vitro in the present study. 


\section{Materials and methods}

Cell culture and identification. Primary human TM cells (cat. no. 634K-05a; Cell Applications, Inc.) were stored in liquid nitrogen $\left(-196^{\circ} \mathrm{C}\right)$. Following resuscitation, the cells were cultured in Dulbecco's modified Eagle's medium (DMEM) (cat. no. 11966025; Gibco; Thermo Fisher Scientific, Inc.) containing 10\% fetal bovine serum (FBS; cat. no. SH30084.03, HyClone; Cytiva) at $37^{\circ} \mathrm{C}$ in a $5 \% \mathrm{CO}_{2}$ cell incubator and $\mathrm{TM}$ cells from passages 3-4 were used for subsequent experiments.

Glucocorticoid induction of myocilin (MYOC) is a method to identify TM cells (11). TM cells were seeded at a density of $1 \times 10^{5}$ cells per well in six-well culture plates and allowed to attach at $37^{\circ} \mathrm{C}$ overnight. The medium was changed the next day for fresh medium containing $100 \mathrm{nM}$ dexamethasone (cat. no. D1756-25MG; MilliporeSigma) or vehicle control ( $1 \%$ ethanol), and the cells were incubated at $37^{\circ} \mathrm{C}$ for another 5 days $(11,12)$. Subsequently, the cells were harvested and total RNA was extracted. The expression level of the MYOC gene was determined in each sample using reverse transcription-quantitative PCR (RT-qPCR). GAPDH was used as the internal control.

Construction of SPARC suppressor gene-containing lentiviral vectors. Viral vectors were constructed using $293 \mathrm{~T}$ cells (cat. no. CRL-11268; American Type Culture Collection). The 293T cells were cultured at $37^{\circ} \mathrm{C}$ in Opti-MEM (cat. no. 31985; Gibco; Thermo Fisher Scientific, Inc.) supplemented with 10\% FBS.

TM cells were infected with different titers [multiplicity of infection (MOI) 0, 20, 50 and 100] of empty control lentiviruses to assess infection efficiency. The infected cells were observed and counted under light (cat. no. CX43; Olympus Corporation; magnification, x200) and fluorescence microscopy (cat. no. IX51; Olympus Corporation; magnification, $\mathrm{x} 200$ ).

The whole sequence of SPARC was acquired from The National Center for Biotechnology Information database (accession no. NM_003118.3, ncbi.nlm.nih. gov/genome/gdv/browser/gene/?id=6678). The oligonucleotides were designed by Virus Lab of Jiao Tong University (Shanghai, China). Three pairs of oligonucleotides were designed and synthesized according to the sequence of SPARC before annealing. The annealed products were cloned into the empty vector PDS134_pL_shRNA_mKate2 (Shanghai Nuobai Biotechnology Co., Ltd.). Thus, three interfering vectors were obtained, including pL_shRNA mKate2-SPARC-543, pL_shRNA_mKate2-SPARC-582 and pL_shRNA_mKate2-SPARC-347 (Table I). The sequences of these vectors were confirmed by Single Molecule Real-Time sequencing (PacBio Sequel; Pacific Biosciences of California).

Subsequently, the 293T cells were co-transfected with the three types of interfering lentiviral vectors separately and the ratio of the lentiviral vector plasmid and packaging plasmid and envelope plasmid was 3:2:1. Lentiviruses were produced using a second-generation packaging system (13). Briefly, 6x10 $293 \mathrm{~T}$ cells were plated in a $15-\mathrm{cm}$ dish and transfected with $9 \mu \mathrm{g}$ Packaging Mix (cat. no. K4975-00; Invitrogen; Thermo Fisher Scientific, Inc.), $3 \mu \mathrm{g}$ transfer plasmid and $36 \mu 1$ Lipofectamine 2000 (cat. no. 11668019; Invitrogen; Thermo Fisher Scientific,
Inc.), which were combined in $5 \mathrm{ml}$ Opti-MEM. The transfection mix was removed once cells were cultured for $6 \mathrm{~h}$ at $37^{\circ} \mathrm{C}$ and fresh Opti-MEM supplemented with $10 \%$ FBS was added. Virus-containing medium was collected at $48 \mathrm{~h}$ post-transfection. Subsequently, the collected medium was filtered through a cellulose acetate membrane (pore size, $0.45 \mathrm{~mm}$ ). The viral stock was collected and concentrated via ultracentrifugation $\left(82,700 \mathrm{x}\right.$ g for $2 \mathrm{~h}$ at $\left.4^{\circ} \mathrm{C}\right)$. Viral titers were measured using flow cytometry (On-Chip Sort; On-chip Biotechnologies Co., Ltd.).

TM cells were infected with the three types of packaged lentiviruses at an MOI of 50. After $24 \mathrm{~h}$ of incubation, the infection efficiency was determined by fluorescence microscopy. The relative expression of SPARC mRNA in TM cells after viral infection were determined using RT-qPCR, as aforementioned.

$m R N A$ and protein expression levels of SPARC, F-actin and ZO-1 following downregulation of SPARC. TM cells at a confluence of $\sim 80 \%$ were selected for this test. The culture medium was removed, and the cells were rinsed with PBS (25X; cat. no. ab64026; Abcam) twice before exposure to serum-free DMEM. The cells were then divided into three groups: i) The blank control group (HTMC group); ii) the empty vector negative control (NC) group (HTMC-NC group), in which target cells were infected with the NC lentivirus (PDS134_pL_shRNA_mKate2); and iii) the lentivirus group (HTMC-SPARC group), in which target cells were infected with pL_shRNA_mKate2-SPARC-543 lentivirus, which demonstrated the greatest efficiency. After $48 \mathrm{~h}$ of culture at $37^{\circ} \mathrm{C}$, the mRNA levels of SPARC, F-actin and ZO-1 were determined using RT-qPCR. The protein levels of SPARC, F-actin and ZO-1 were determined using western blotting. GAPDH was used as the internal control.

Escherichia coli phagocytosis experiment in TM cells. TM cells at a confluence of $\sim 50 \%$ were selected for this test. The culture medium was removed, and the cells were rinsed with PBS twice before exposure to DMEM containing 10\% FBS. The cells were then divided into 3 groups: HTMC group, the HTMC-NC group and the HTMC-SPARC group. Subsequently, $0.1 \mathrm{ml}$ inactivated Escherichia coli suspension $\left(1.6 \times 10^{9} \mathrm{CFU} / \mathrm{ml}\right)$ was added to the cells. After culture for another 24 or $48 \mathrm{~h}$, the culture medium was removed and the cells were rinsed with PBS twice before extraction of RNA. RT-qPCR was used to determine the expression of bacterial 16S RNA in the cells to evaluate phagocytosis, as aforementioned.

$R T-q P C R$. Human TM cells were harvested and total RNA was extracted. The expression levels of MYOC, GAPDH, SPARC, ZO-1, F-actin, and 16S were detected. Total RNA was extracted using TRIzol ${ }^{\circledR}$ (cat. no. 15596018; Invitrogen; Thermo Fisher Scientific, Inc.) according to the manufacturer's instructions. A SuperScript First-Strand Synthesis system (cat. no. 11904-018; Invitrogen; Thermo Fisher Scientific, Inc.) was used for RT. Each reaction contained $0.5 \mu \mathrm{l}$ random primers $(0.2 \mu \mathrm{g} / \mu \mathrm{l})$ and $1.0 \mu \mathrm{l}$ SuperScript III reverse transcriptase $(200 \mathrm{U} / \mu \mathrm{l})$. The specific primers used are listed in Table II. qPCR was performed using the SYBR ${ }^{\circledR}$ Premix Ex Taq (cat. no. RR420L; Takara Bio, Inc.). The thermocycling conditions were as follows: Initial 
Table I. Construction of SPARC suppressor gene-containing lentiviral vectors.

\begin{tabular}{ll} 
shRNA & \multicolumn{1}{c}{ Sequences (5'-3') } \\
\hline $\begin{array}{l}\text { pL_shRNA_mKate2-SPARC-543 } \\
\text { Forward }\end{array}$ & CACCGGATGAGGACAACAACCTTCTCGAAAGAAGGTTGTTGTCCTCATCC \\
Reverse & AAAAGGATGAGGACAACAACCTTCTTTCGAGAAGGTTGTTGTCCTCATCC \\
pL_shRNA_mKate2-SPARC-582 & \\
Forward & CACCGCGGGTGAAGAAGATCCATGACGAATCATGGATCTTCTTCACCCGC \\
Reverse & AAAAGCGGGTGAAGAAGATCCATGATTCGTCATGGATCTTCTTCACCCGC \\
pL_shRNA_mKate2-SPARC-347 & \\
Forward & CACCGCCAGGTGGAAGTAGGAGAATTCGAAAATTCTCCTACTTCCACCTGG \\
Reverse & AAAACCAGGTGGAAGTAGGAGAATTTTCGAATTCTCCTACTTCCACCTGGC
\end{tabular}

shRNA, short hairpin RNA; SPARC, secreted protein acidic and rich in cysteine.

denaturation at $95^{\circ} \mathrm{C}$ for $2 \mathrm{~min}$; denaturing at $95^{\circ} \mathrm{C}$ for $10 \mathrm{sec}$; annealing at $60^{\circ} \mathrm{C}$ for $30 \mathrm{sec}$ and polymerization at $70^{\circ} \mathrm{C}$ for 45 sec. A total of 40 PCR cycles was performed. PCR was performed using a CFX96 Touch Real-Time PCR Detection system. Gene expression was determined as the ratio of relative optical density of target gene to GAPDH. The $2^{-\Delta \Delta \mathrm{Cq}}$ method was utilized to measure PCR results (14).

Western blot analysis. Protein expression levels of SPARC, F-actin and ZO-1 in TM cells were detected using western blot analysis. The cells were lysed in lysis buffer (cat. no. P0013, Beyotime Institute of Biotechnology) at $4^{\circ} \mathrm{C}$ with phosphatase and protease inhibitors (cat. no. P1045; Beyotime Institute of Biotechnology). The lysis mixture was centrifuged at $4^{\circ} \mathrm{C}$ for $10 \mathrm{~min}$ at $10,000 \mathrm{xg}$, and the supernatant containing cellular proteins was utilized for subsequent experiments. The protein concentration was measured using a BCA kit. Proteins were separated using SDS-PAGE (10\% gel; $40 \mu \mathrm{g} /$ lane; $120 \mathrm{~V})$. The separated proteins were then transferred to polyvinylidene fluoride membranes (100 V for $120 \mathrm{~min}$; cat. no. FFP24; Beyotime Institute of Biotechnology). The membranes were blocked with 5\% non-fat milk at room temperature for $1 \mathrm{~h}$ and incubated with anti-SPARC (400 $\mu \mathrm{l}$; cat. no. ab51399; Abcam; 1:1,000), anti-ZO-1 (50 $\mu$ g; cat. no. ab59720, Abcam; 1:250), or anti-F-actin (cat. no. ab205, Abcam; 1:1,000) primary antibodies at $4^{\circ} \mathrm{C}$ overnight. Membranes were washed with Tris-buffered saline containing $0.1 \%$ Tween-20 and incubated with horseradish peroxidase-conjugated secondary antibodies (goat anti-chicken IgY, anti-rabbit IgG and anti-mouse IgM; all 1:2,000; cat. nos. ab6877, ab6721 and ab47827; all Abcam) at room temperature for $1 \mathrm{~h}$. Membranes were incubated in enhanced chemiluminescence solution (cat. no. P0018A; Beyotime Institute of Biotechnology). Images were captured on film (cat. no. FF057, Beyotime Institute of Biotechnology) in a dark room. Experiments were repeated three times. Blot images were quantified in greyscale using ImageJ (version 1.5.2; National Institutes of Health).

Flow cytometry to titrate lentiviral vectors. The cells were seeded at a density of $5 \times 10^{4}$ cells/well in a 6-well plate. At $24 \mathrm{~h}$ post-seeding, the number of cells was counted in two wells using a hemacytometer. The medium in other wells was replaced with
$0.5 \mathrm{ml}$ fresh Opti-MEM supplemented with $10 \%$ FBS containing $8 \mathrm{mg} / \mathrm{ml}$ polybrene. Next, the cells were transduced by adding $0.5,5.0$ and $50.0 \mathrm{ml}$ aliquots vector stock/well and placed back in the incubator at $37^{\circ} \mathrm{C}$. After $20 \mathrm{~h}$, the medium was replaced with $2 \mathrm{ml}$ fresh medium and incubated at $37^{\circ} \mathrm{C}$ for another 2 days, Then, the medium was removed and cells were washed with $1 \mathrm{ml}$ PBS. After that, $0.5 \mathrm{ml} /$ well trypsin-EDTA was added and the cells were incubated at $37^{\circ} \mathrm{C}$ for $2 \mathrm{~min}$. Next, $1 \mathrm{ml}$ medium was added to each well and contents were mixed. The cell suspension was transferred into a 5-ml round-bottomed tube and centrifuged at $500 \mathrm{x} \mathrm{g}$ for $5 \mathrm{~min}$ at $20^{\circ} \mathrm{C}$. The medium was removed and cells were resuspend in $2 \mathrm{ml}$ Hank's balanced salt solution and centrifuged at $500 \mathrm{x} \mathrm{g}$ for $5 \mathrm{~min}$ at $20^{\circ} \mathrm{C}$. Subsequently, Hank's balanced salt solution was removed and the cells were resuspend in $300 \mathrm{ml}$ Hank's balanced salt solution. Then, the cells were analyzed using a flow cytometer and titers were calculated (15).

Statistical analysis. All the experiments were repeated in triplicate. The statistical analysis was performed using SPSS 19.0 software (IBM Corp.). Data are presented as the mean \pm standard deviation. Differences between two groups were measured using Student's t-test (paired). Differences among multiple groups were measured using Student Newman Keuls test or two-way ANOVA followed by Tukey's post hoc. $\mathrm{P}<0.05$ was considered to indicate a statistically significant difference.

\section{Results}

MYOC expression after dexamethasone exposure. After 5 days of exposure to $100 \mathrm{nM}$ dexamethasone, the TM cells in the dexamethasone group exhibited a mean 7.62-fold increase in MYOC gene expression compared with those in vehicle control group (Fig. 1). This indicated that the MYOC could be induced by glucocorticoids in this commercial human TM cell strain and that this cell strain met the needs of subsequent experiments.

Construction of SPARC suppressor gene-containing lentiviral vectors. TM cells were infected with different titers (MOI 0 , 20, 50 and 100) of empty control lentiviruses to assess infection efficiency. It was revealed that 80.2 and $89.7 \%$ of TM cells were infected with empty control lentiviruses at MOIs of 50 and 
Table II. Primers used in reverse transcription-quantitative PCR.

\begin{tabular}{ll}
\hline Primer & \multicolumn{1}{c}{ Sequence, ${\text { 5' } \rightarrow 3^{\prime}}^{\prime}$} \\
\hline $\begin{array}{l}\text { MYOC } \\
\text { Forward } \\
\text { Reverse } \\
\text { GAPDH }\end{array}$ & TACCGAGACAGTGAAGGCTG \\
Forward & TGTAGCTGCTGACGGTGTAC \\
Reverse & CGTATTGGGCGCCTGGTCACC \\
SPARC & GGGATGATGTTCTGGAGAGCCC \\
Forward & AGGAAACCGAAGAGGAGG \\
Reverse & GCAAAGAAGTGGCAGGAA \\
ZO-1 & \\
Forward & TATTCACGCAGTTACGAGCAAG \\
Reverse & AAGGTATCAGCGGAGGGACA \\
F-actin & \\
Forward & GTCACCAACTGGGACGACA \\
Reverse & CACAGCCTGGATAGCAACG \\
$16 S$ & \\
Forward & CCGCATAATGTCGCAAGACC \\
Reverse & TCAGACCAGCTAGGGATCGT \\
\hline
\end{tabular}

MYOC, myocilin; SPARC, secreted protein acidic and rich in cysteine; ZO-1, zonula occludens- 1 .

100 , respectively, which was satisfactory infection efficiency for subsequent experiments, as revealed by non-fluorescence microscopy and fluorescence microscopy (Fig. 2A and B). To avoid cell physiological dysfunction or death, MOI of 50 was selected for subsequent experiments.

Three pairs of oligonucleotides were designed according to the sequence of SPARC. The annealed products were cloned into the empty vector PDS134_pL_shRNA_mKate2. The 293T cells were co-transfected with the three types of interfering lentiviral vectors separately and lentiviruses were produced using a second-generation packaging system. The fluorescence ratio was determined to calculate viral titers, which were revealed to be similar among the three types of lentiviruses (Table III).

Subsequently, TM cells were infected with the three types of packaged lentiviruses at an MOI of 50. After $24 \mathrm{~h}$ of incubation, the mean infection efficiency was 79.2, 82.1 and $74.0 \%$ in pL_shRNA_mKate2-SPARC-543, pL_shRNA mKate2-SPARC-582 and pL_shRNA_mKate2-SPARC-347, respectively, which were showed that all three lentiviruses achieved satisfactory results, as revealed by fluorescence microscopy (Fig. 3).

The relative expression levels of SPARC mRNA in TM cells after viral infection were determined using RT-qPCR. The mean interference efficiency of pL_shRNA mKate2-SPARC-543, pL_shRNA_mKate2-SPARC-582 and pL_shRNA_mKate2-SPARC-347 was 74.2, 36.0 and $62.5 \%$, respectively. The pL_shRNA_mKate2-SPARC-543 had a significantly decreased expression level of SPARC compared

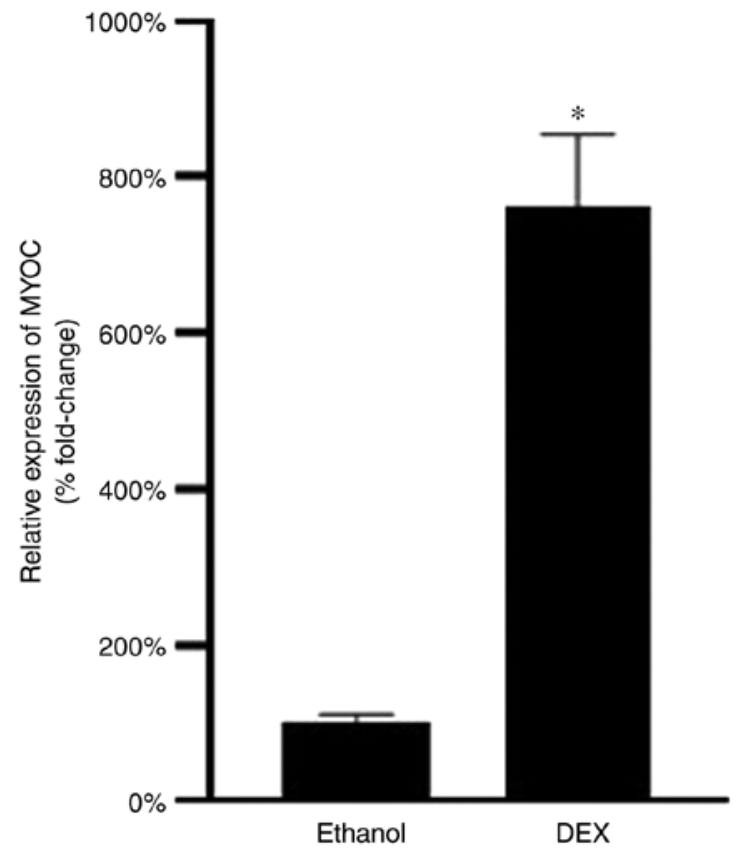

Figure 1. Expression levels of MYOC in HTMCs with or without dexamethasone exposure. HTMCs were cultured with $100 \mathrm{nM}$ dexamethasone or vehicle control ( $1 \%$ ethanol), incubated for 5 days and then harvested for total RNA. Expression levels of the MYOC gene were determined in each sample using reverse transcription-quantitative PCR. GAPDH was used as the internal control. ${ }^{*} \mathrm{P}<0.05$ vs. vehicle control group. DEX, dexamethasone; MYOC, myocilin; HTMC, human trabecular meshwork cell.

with the NC; it was also the most efficient in interfering with SPARC mRNA expression in human TM cells and, therefore, it was used for subsequent experiments (Fig. 4).

Effects of SPARC downregulation on F-actin and ZO-1 expression. The mRNA expression levels of SPARC, F-actin and ZO-1 were analyzed after $48 \mathrm{~h}$ of culture in the empty vector control (HTMC-NC) group, which was used to represent $100 \%$ expression. The relative mRNA expression levels of SPARC, F-actin and ZO-1 were 107, 118 and 93\% in the blank control (HTMC) group and 39,52 and 52\% in the lentivirus (HTMC-SPARC-543) group, respectively. RT-qPCR revealed that the mRNA expression levels of SPARC, F-actin and ZO-1 were significantly lower in the HTMC-SPARC-543 group compared with the levels in the HTMC and HTMC-NC groups (Fig. 5A).

The protein expression levels of SPARC, F-actin and ZO-1 were analyzed and the empty vector control (HTMC-NC) groups were used to represent $100 \%$ expression. The relative protein expression levels of SPARC, F-actin and ZO-1 were 94, 95 and $110 \%$ in the blank control (HTMC) group and 34,44 and $38 \%$ in the lentivirus (HTMC-SPARC-543) group, respectively. The western blotting results also revealed that the protein expression levels of SPARC, F-actin and ZO-1 were significantly lower in the HTMC-SPARC-543 group compared with those in the HTMC and HTMC-NC groups (Fig. 5B and C). Overall, these results demonstrated that downregulation of SPARC significantly decreased the expression of F-actin and ZO-1.

Effects of SPARC downregulation on phagocytosis. Research has indicated that phagocytosis in TM cells is active, 
A

\section{uorescer Microscopy}

20
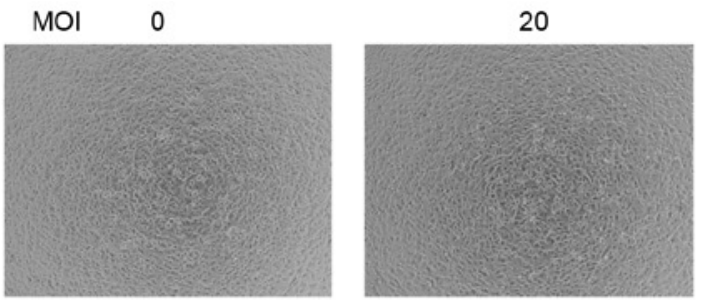

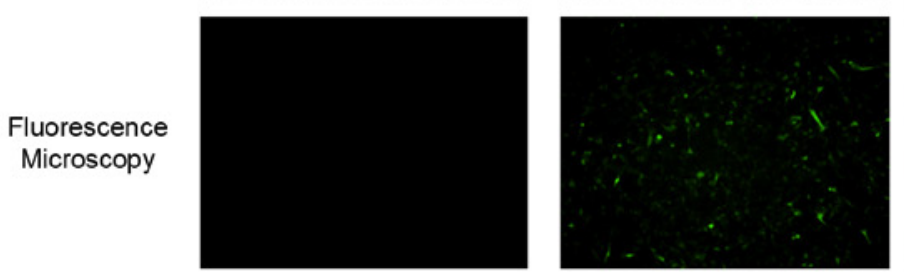

B $100 \%$
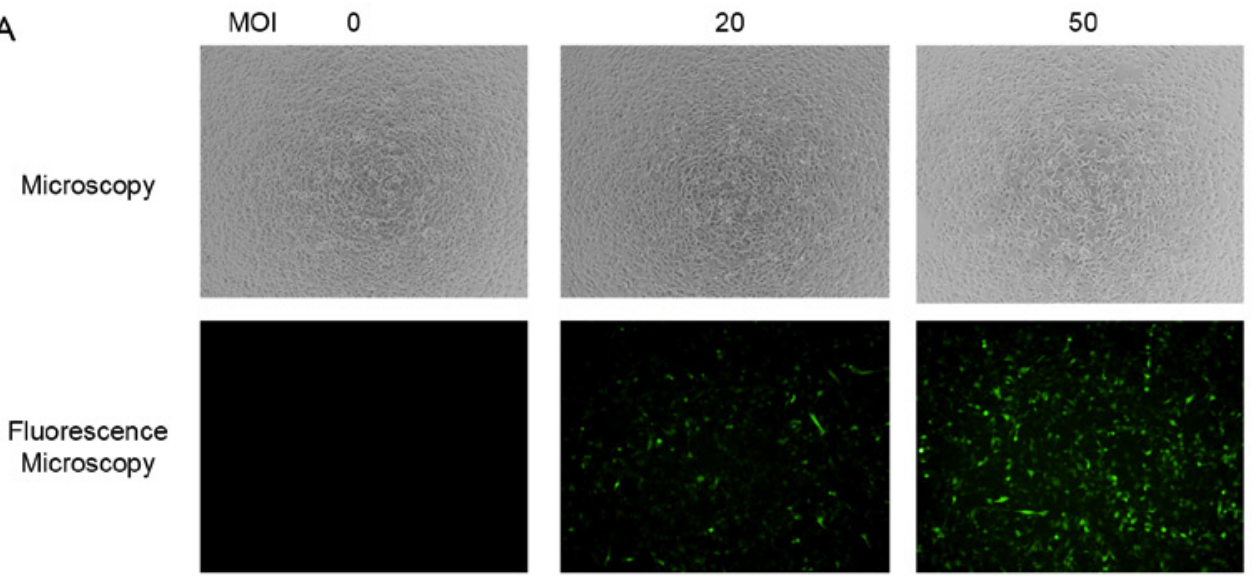

100
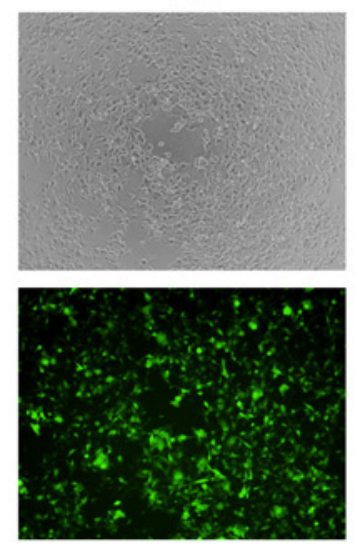

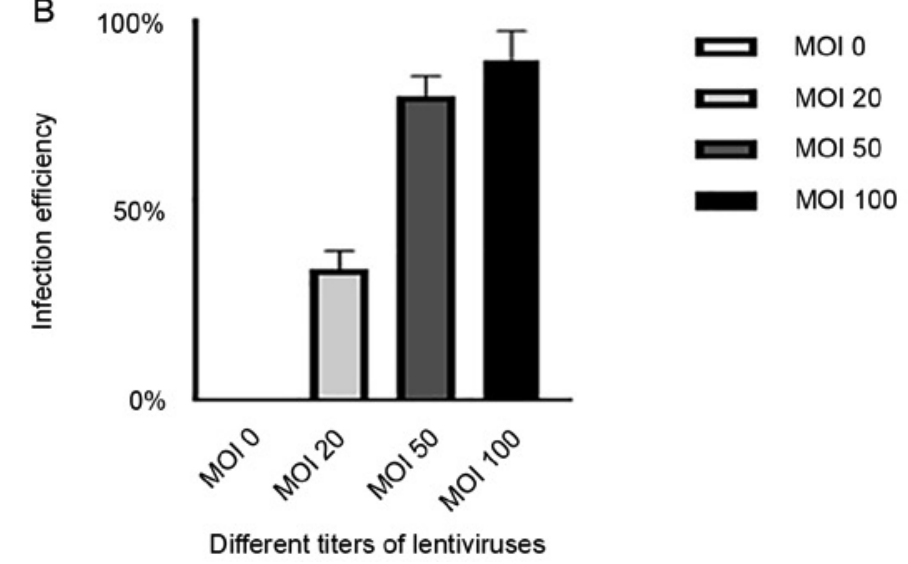

Figure 2. Infection efficiency of different empty control lentiviruses titers. Trabecular meshwork cells were infected with different titers (MOI 0, 20, 50 and 100 ) of empty control lentiviruses to assess infection efficiency. Infected cells were (A) observed and (B) counted under non-fluorescence microscopy and fluorescence microscopy. Magnification, x200. MOI, multiplicity of infection.

especially in the uveal and corneoscleral meshwork (11). Shirato et al (16) demonstrated that phagocytosis reached half-maximum at $40 \mathrm{~h}$ and maximum at $96 \mathrm{~h}$ in TM cells in vitro; therefore 24 and $48 \mathrm{~h}$ were selected as observational time points in the present study. An equal amount of inactivated Escherichia coli suspension was added to each group. After 24 or $48 \mathrm{~h}$ of culture, the expression levels of bacterial 16S RNA in TM cells were detected by RT-qPCR. The expression of 16S RNA in the HTMC-NC group after 5 min of culture was used as a control to represent $100 \%$ expression. Thus, the relative expression levels in the HTMC-NC, HTMC and SPARC-543 groups were 278, 272 and 403\% after $24 \mathrm{~h}$ of culture, and 283, 274 and $421 \%$ after $48 \mathrm{~h}$ of culture, respectively. The expression levels of 16S RNA were significantly increased in the SPARC-543 groups compared with those in the HTMC-NC and HTMC groups. However, the expression of 16S RNA in these three groups did not differ between 24 and $48 \mathrm{~h}$ of culture (Fig. 6). These results indicated that SPARC downregulation significantly promoted the phagocytosis of TM cells and that phagocytosis of TM cells reached a peak within $24 \mathrm{~h}$.

\section{Discussion}

IOP, as determined by the production, circulation and drainage of aqueous humor, is the main risk factor for glaucoma. The trabecular outflow pathway via which aqueous humor passes through the TM into Schlemm's canal, known as the conventional outflow pathway, serves a key function in maintaining the IOP. TM cells are the primary cell type that occupy and form this outflow pathway, in coordination with the inner wall of Schlemm's canal. Cellular dysfunction of TM cells results in the generation of extra resistance that causes elevated IOP. To ensure effective regulation of outflow resistance, TM tissue has two primary responsibilities, namely, filtration and resistance generation (11). Accordingly, TM cells display different morphologies and characteristics to support these two primary responsibilities (11).

TM cells in the inner TM tissue, the uveal meshwork and the corneoscleral meshwork have macrophage-like activity and act as professional endothelial cells. Phagocytosis is an essential function of TM cells in this role (11). Notably, rapid clearance of debris by the inner TM occurs prior to it reaching into the deep TM where it could accumulate and disrupt resistance generation and regulation (11). Cultured TM cells and TM cells in vivo are actively phagocytic, and this is an important part of maintaining a clean outflow filter (17). TM cell phagocytosis changes in response to environmental changes. A series of studies have indicated that TM cell phagocytosis can be inhibited by dexamethasone (18), $\gamma$-interferon (19), epinephrine and cortisone (20), and that impaired TM cell phagocytosis may be one of the pathogeneses of some types of glaucoma. By contrast, promoting phagocytosis shows several prospects for clinical application. The results of the present 
Table III. Fluorescence ratio of the three viral titers.

\begin{tabular}{lccc}
\hline & & Fluorescence ratio, & Viral titers, \\
Virus & $\mu 1$ & $\%$ & TU/ml \\
\hline pL_shRNA_mKate2-SPARC-543 & 1 & 100 & $2.0 \times 10^{8}$ \\
pL_shRNA_mKate2-SPARC-582 & 1 & 90 & $1.8 \times 10^{8}$ \\
pL_shRNA_mKate2-SPARC-347 & 1 & 80 & $1.6 \times 10^{8}$ \\
\hline
\end{tabular}

shRNA, short hairpin RNA; SPARC, secreted protein acidic and rich in cysteine.

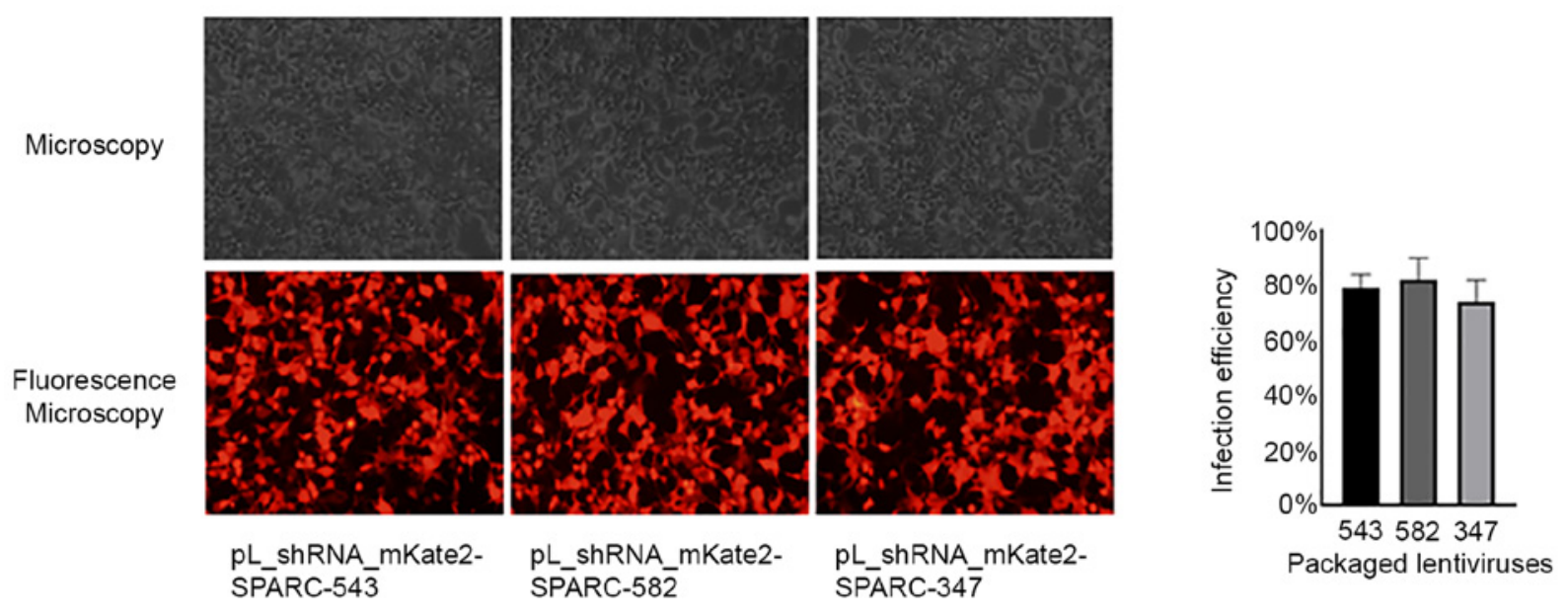

Figure 3. Transfection efficiency of the three types of lentiviruses. Trabecular meshwork cells were transfected with the three types of packaged lentiviruses at a multiplicity of infection of 50. After $24 \mathrm{~h}$ of incubation, transfection efficiency was revealed to be desirable for all the three types of lentiviruses, as revealed by fluorescence microscopy. Magnification, x200. 543, pL_shRNA_mKate2-SPARC-543 group; 582, pL_shRNA_mKate2-SPARC-582 group; 347, pL_shRNA_mKate2-SPARC-347 group; SPARC, secreted protein acidic and rich in cysteine; shRNA, short hairpin RNA.

study demonstrated that SPARC downregulation significantly increased the phagocytosis of TM cells and contributed to aqueous humor outflow. Although the mechanism remains to be clarified, SPARC downregulation shows promise for application as an anti-glaucoma treatment, especially for pseudoexfoliation syndrome or pigmented glaucoma, in which large amounts of pigment granules and debris are produced.

TM cells in the outer TM tissue, the JCT region, have both fibroblastic and smooth muscle-like qualities (11). Previous research has demonstrated that fibrosis of the JCT is a major cause of impaired aqueous humor outflow and glaucoma (21). The mechanical and biological changes associated with JCT fibrosis, in turn, are caused by structural changes in the cytoskeleton (22) and ECM (23). These structural changes are considered upstream signals in the pathological process of glaucoma (24). The present study indicated that downregulation of SPARC lowered the expression of F-actin in TM cells, leading to rearrangement of the actin cytoskeleton. F-actin in the cytoskeleton is one of the key factors that determines cell mechanics (25). F-actin morphology is closely associated with functional disorders of TM cells $(26,27)$. The cytoskeleton plays notable roles in maintaining cell polarity and initiating intracellular metabolism and signaling $(28,29)$. Cytoskeletal rearrangement is also closely associated with phagocytosis, cell secretion $(30,31)$, cell junction changes and changes in the composition of the ECM $(32,33)$. In addition, the distribution and structure of the cytoskeleton directly affects contraction of the TM (28). These factors can directly or indirectly change the outflow resistance of aqueous humor.

The results of the present study also indicated that SPARC downregulation led to decreased expression of ZO-1, causing F-actin to detach from the cell membrane. ZO-1 plays a central role in cytoskeleton-associated proteins; it is attached to the cell membrane, with one end linked to occludin, a tight junction protein in the cell membrane, and the other end linked to F-actin in the cytoplasm. Together, these proteins together form a functional complex that is the main permeability barrier among cells. The barrier regulates intercellular transport of water, ions and macromolecules, bridges internal and external cell communication, modulates cell movement and maintains the cell microenvironment (33). A previous study has demonstrated that changes in ZO-1 expression or location and/or structural dysfunction of ZO-1 may disrupt the integrity of tight junctions, resulting in loss of intercellular junctions and impaired permeability (34). $\mathrm{ZO}-1$ is involved in cell and tissue remodeling. ZO-1-deficient mice exhibit delayed growth (35). Previous research has demonstrated that ZO-1 is also involved in the regulation of TM function. Increased expression of ZO-1 in TM cells has been noted in patients with neovascular glaucoma (36). Increased IOP can lead to decreased expression of ZO-1 and affect the cytoskeleton of TM cells and intercellular adhesion (37). Dexamethasone increases the protein expression level of ZO-1 in cultured TM cells (38). These results indicate that ZO-1 is closely associated 


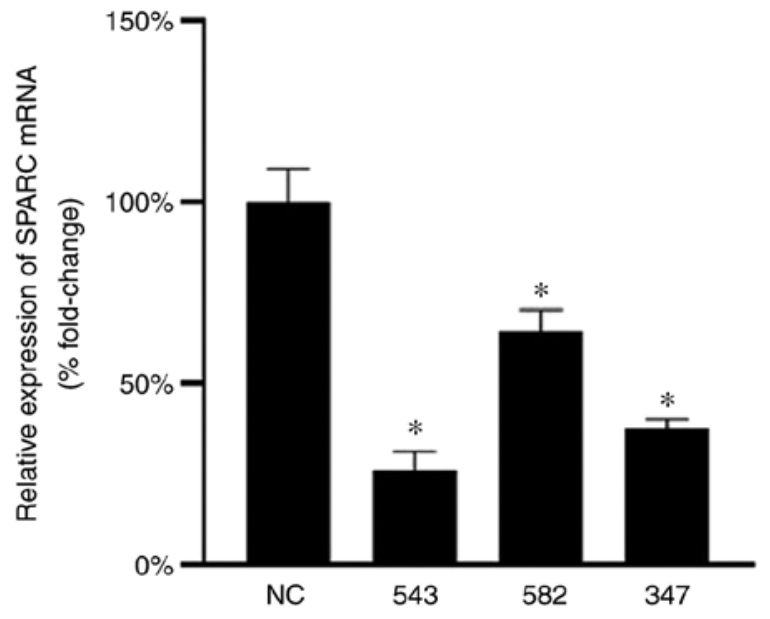

Figure 4. Expression of SPARC mRNA in HTMCs after viral infection. Relative expression levels of SPARC mRNA in HTMCs after viral infection were determined using reverse transcription-quantitative PCR. ${ }^{*} \mathrm{P}<0.05$ vs. NC group. NC, negative control; 543, pL_shRNA_mKate2-SPARC-543 group; 582, pL_shRNA_mKate2-SPARC-582 group; 347, pL_shRNA_ mKate2-SPARC-347 group; HTMC, human trabecular meshwork cell; SPARC, secreted protein acidic and rich in cysteine.

with cytoskeletal rearrangement and cellular contractile tone, therefore affecting the drainage of the aqueous humor, and that SPARC may initiate this process.

In human eyes, SPARC is present in numerous tissues, including the lens (39), corneal epithelium (39), TM cells (3), ciliary body smooth muscle cells (40), aqueous and vitreous humors (39), and retinal pigment epithelium $(39,41)$. Immunofluorescence staining revealed that SPARC is present throughout the TM and that it is among the most abundantly expressed genes in cultured human TM cells $(4,42)$. In the TM of human eyes at postmortem, SPARC and MYOC, another glaucoma gene, increased significantly with increased levels of IOP (43). SPARC-null mice were demonstrated to have lower IOP compared with wild-type mice, potentially due to decreased outflow resistance (5), and TGF- $\beta 2$ failed to induce ocular hypertension in such mice (44). These results indicate that SPARC is important in the regulation of aqueous humor outflow via the TM, although the underlying mechanism remains unclear. The results of the present study suggested that SPARC may work throughout the whole TM tissue by a variety of mechanisms, including improving phagocytosis and cytoskeletal rearrangement.

The current study used only one commercial human TM cell strain to test MYOC as the cell marker. By contrast, directly acquiring cells from human TM tissues would increase the validity of the results. Meanwhile, precise cell identification is indispensable in primary cells. Furthermore, in vivo tests are needed to validate the conclusions the present study revealed in vitro. The mechanism whereby SPARC regulates IOP, the signaling pathways upstream of SPARC and its regulation of ECM metabolism represent future research directions in this field. We consider that SPARC will hold promise as a novel therapeutic target for glaucoma as more information is revealed on this matricellular protein.

In conclusion, downregulation of SPARC decreased the expression levels of the cytoskeleton-associated proteins F-actin and ZO-1, promoted phagocytosis in human TM cells

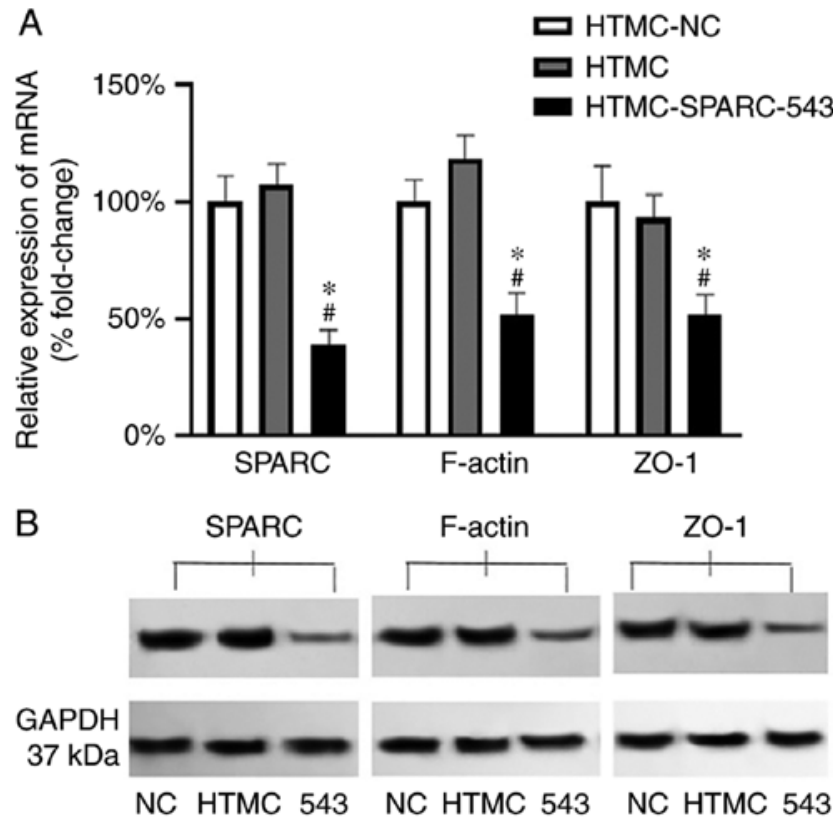

C Relative protein expression

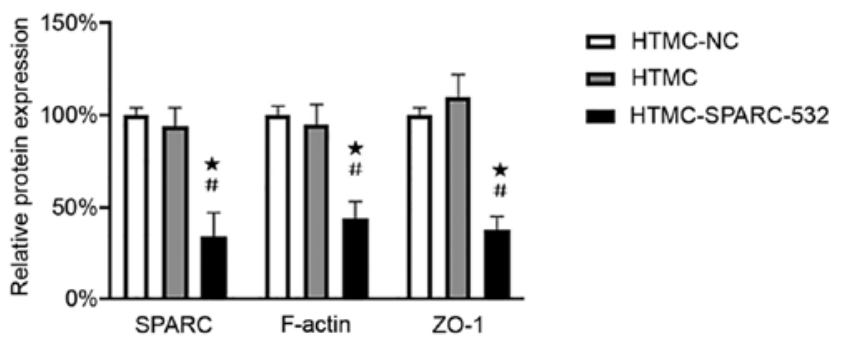

Figure 5. Effects of SPARC downregulation on F-actin and ZO-1 expression in HTMCs. HTMCs were divided into three groups: Empty vector control group (HTMC-NC group), blank control group (HTMC group) and the lentivirus group (HTMC-SPARC group). (A) After $48 \mathrm{~h}$ of culture, the mRNA expression levels of SPARC, F-actin and ZO-1 were determined using reverse transcription-quantitative PCR. Protein expression levels of SPARC, F-actin and ZO-1 were determined using (B) western blotting and (C) quantified. ${ }^{*} \mathrm{P}<0.05$ vs. the HTMC-NC group; ${ }^{~} \mathrm{P}<0.05$ vs. the HTMC group. SPARC, secreted protein acidic and rich in cysteine; ZO-1, zonula occludens-1; HTMC, human trabecular meshwork cell; NC, negative control; 543,pL_shRNA_mKate2-SPARC-543 group.

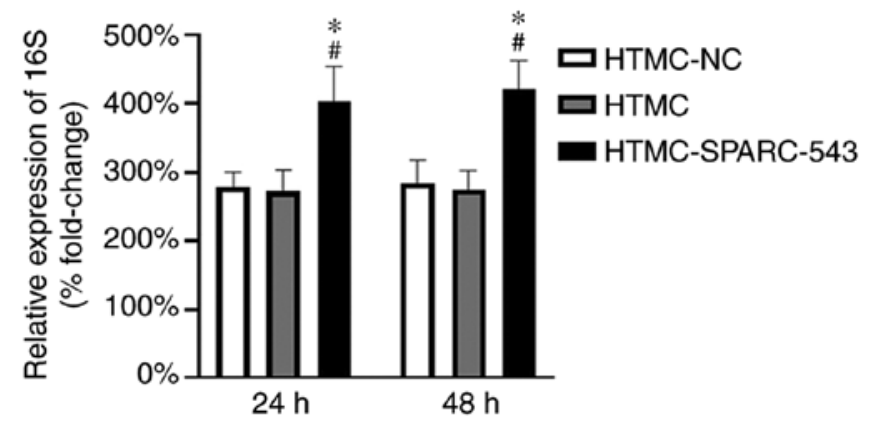

Figure 6. Effects of SPARC downregulation on phagocytosis in HTMCs. An equal amount of inactivated Escherichia coli suspension was added to the HTMC-NC, HTMC and HTMC-SPARC groups. After 24 or $48 \mathrm{~h}$ of culture, the expression levels of bacterial 16S RNA in TM cells were detected by reverse transcription-quantitative PCR, and 16S RNA in the HTMC-NC group after 5 min of culture was used as a control to represent $100 \%$ expression. ${ }^{*} \mathrm{P}<0.05$ vs. the HTMC-NC group; ${ }^{\# P}<0.05$ vs. the HTMC group. SPARC, secreted protein acidic and rich in cysteine; HTMC, human trabecular meshwork cell; NC, negative control; 543, pL_shRNA_mKate2-SPARC-543 group. 
and may affect the outflow of aqueous humor through the TM pathway.

\section{Acknowledgements}

Not applicable.

\section{Funding}

No funding was received.

\section{Availability of data and materials}

The datasets used and/or analyzed during the current study are available from the corresponding author on reasonable request.

\section{Authors' contributions}

YaF, LL, YiF and MT designed the experimental protocol. MT contributed to the study design. LL performed the experimental procedures. YiF drafted the paper. YiF and MT performed the critical revision of the manuscript. All authors have read and approved the final version of the manuscript. YiF and MT confirm the authenticity of all the raw data.

\section{Ethics approval and consent to participate}

This study was approved by The Ethics Committee of Shanghai General Hospital, Shanghai Jiao Tong University (Shanghai, China; approval no. 2016KY034).

\section{Patient consent for publication}

Not applicable.

\section{Competing interests}

The authors declare that they have no competing interests.

\section{References}

1. Tham YC, Li X, Wong TY, Quigley HA, Aung T and Cheng CY: Global prevalence of glaucoma and projections of glaucoma burden through 2040: A systematic review and meta-analysis. Ophthalmology 121: 2081-2090, 2014.

2. Bourne RR, Taylor HR, Flaxman SR, Keeffe J, Leasher J, Naidoo K, Pesudovs K, White RA, Wong TY, Resnikoff S, et al: Number of people blind or visually impaired by glaucoma worldwide and in world regions 1990-2010: A meta-analysis. PLoS One 11: e0162229, 2016.

3. Rhee DJ, Fariss RN, Brekken R, Sage EH and Russell P: The matricellular protein SPARC is expressed in human trabecular meshwork. Exp Eye Res 77: 601-607, 2003.

4. Vittal V, Rose A, Gregory KE, Kelley MJ and Acott TS: Changes in gene expression by trabecular meshwork cells in response to mechanical stretching. Invest Ophthalmol Vis Sci 46: 2857-2868, 2005.

5. Haddadin RI, Oh DJ, Kang MH, Filippopoulos T, Gupta M, Hart L and Rhee DJ: SPARC-null mice exhibit lower intraocular pressures. Invest Ophthalmol Vis Sci 50: 3771-3777, 2009.

6. Chua J, Seet LF, Jiang Y, Su R, Htoon HM, Charlton A, Aung T and Wong TT: Increased SPARC expression in primary angle closure glaucoma iris. Mol Vis 14: 1886-1892, 2008.

7. Stamer WD: The cell and molecular biology of glaucoma: Mechanisms in the conventional outflow pathway. Invest Ophthalmol Vis Sci 53: 2470-2472, 2012.
8. Tian B, Gabelt BT, Geiger B and Kaufman PL: The role of the actomyosin system in regulating trabecular fluid outflow. Exp Eye Res 88: 713-717, 2009.

9. Bradshaw AD: The role of secreted protein acidic and rich in cysteine (SPARC) in cardiac repair and fibrosis: Does expression of SPARC by macrophages influence outcomes? J Mol Cell Cardiol 93: 156-161, 2016.

10. Nie J and Sage EH: SPARC functions as an inhibitor of adipogenesis. J Cell Commun Signal 3: 247-254, 2009.

11. Stamer WD and Clark AF: The many faces of the trabecular meshwork cell. Exp Eye Res 158: 112-123, 2017.

12. Fan BJ, Wang DY, Tham CC, Lam DS and Pang CP: Gene expression profiles of human trabecular meshwork cells induced by triamcinolone and dexamethasone. Invest Ophthalmol Vis Sci 49: 1886-1897, 2008.

13. Zufferey R, Nagy D, Mandel RJ, Naldini L and Trono D: Multiply attenuated lentiviral vector achieves efficient gene delivery in vivo. Nat Biotechnol 15: 871-875, 1997.

14. Livak KJ and Schmittgen TD: Analysis of relative gene expression data using real-time quantitative PCR and the 2(-Delta Delta C(T)) method. Methods 25: 402-408, 2001.

15. Kutner RH, Zhang XY and Reiser J: Production, concentration and titration of pseudotyped HIV-1-based lentiviral vectors. Nat Protoc 4: 495-505, 2009.

16. Shirato S, Murphy CG, Bloom E, Franse-Carman L, Maglio MT, Polansky JR and Alvarado JA: Kinetics of phagocytosis in trabecular meshwork cells. Flow cytometry and morphometry. Invest Ophthalmol Vis Sci 30: 2499-2511, 1989.

17. Johnson DH, Richardson TM and Epstein DL: Trabecular meshwork recovery after phagocytic challenge. Curr Eye Res 8: 1121-1130, 1989.

18. Zhang X, Ognibene CM, Clark AF and Yorio T: Dexamethasone inhibition of trabecular meshwork cell phagocytosis and its modulation by glucocorticoid receptor beta. Exp Eye Res 84: 275-284, 2007.

19. Park CH and Latina MA: Effects of gamma-interferon on human trabecular meshwork cell phagocytosis. Invest Ophthalmol Vis Sci 34: 2228-2236, 1993.

20. Yang $X$ and Li M: Establishment of in vitro culture of bovine trabecular meshwork cells and their phagocytosis. Zhonghua Yan Ke Za Zhi 32: 136-139, 1996 (In Chinese).

21. Last JA, Pan T, Ding Y, Reilly CM, Keller K, Acott TS, Fautsch MP, Murphy CJ and Russell P: Elastic modulus determination of normal and glaucomatous human trabecular meshwork. Invest Ophthalmol Vis Sci 52: 2147-2152, 2011.

22. Hoare MJ, Grierson I, Brotchie D, Pollock N, Cracknell K and Clark AF: Cross-linked actin networks (CLANs) in the trabecular meshwork of the normal and glaucomatous human eye in situ. Invest Ophthalmol Vis Sci 50: 1255-1263, 2009.

23. Lutjen-Drecoll E: Morphological changes in glaucomatous eyes and the role of TGFbeta2 for the pathogenesis of the disease. Exp Eye Res 81: 1-4, 2005.

24. Morgan JT, Raghunathan VK, Chang YR, Murphy CJ and Russell P: The intrinsic stiffness of human trabecular meshwork cells increases with senescence. Oncotarget 6: 15362-15374, 2015.

25. Chen QM, Tu VC, Catania J, Burton M, Toussaint O and Dilley T: Involvement of $\mathrm{Rb}$ family proteins, focal adhesion proteins and protein synthesis in senescent morphogenesis induced by hydrogen peroxide. J Cell Sci 113: 4087-4097, 2000.

26. Tian B, Geiger B, Epstein DL and Kaufman PL: Cytoskeletal involvement in the regulation of aqueous humor outflow. Invest Ophthalmol Vis Sci 41: 619-623, 2000.

27. Clark AF, Brotchie D, Read AT, Hellberg P, English-Wright S, Pang IH, Ethier CR and Grierson I: Dexamethasone alters F-actin architecture and promotes cross-linked actin network formation in human trabecular meshwork tissue. Cell Motil Cytoskeleton 60: 83-95, 2005.

28. Stumpff F and Wiederholt M: Regulation of trabecular meshwork contractility. Ophthalmologica 214: 33-53, 2000.

29. Kaufman PL: Enhancing trabecular outflow by disrupting the actin cytoskeleton, increasing uveoscleral outflow with prostaglandins, and understanding the pathophysiology of presbyopia interrogating Mother Nature: Asking why, asking how, recognizing the signs, following the trail. Exp Eye Res 86: $3-17,2008$

30. Sanka K, Maddala R, Epstein DL and Rao PV: Influence of actin cytoskeletal integrity on matrix metalloproteinase-2 activation in cultured human trabecular meshwork cells. Invest Ophthalmol Vis Sci 48: 2105-2114, 2007. 
31. Gilles C, Bassuk JA, Pulyaeva H, Sage EH, Foidart JM and Thompson EW: SPARC/osteonectin induces matrix metalloproteinase 2 activation in human breast cancer cell lines. Cancer Res 58: 5529-5536, 1998.

32. Liu X, Wu Z, Sheibani N, Brandt CR, Polansky JR and Kaufman PL: Low dose latrunculin-A inhibits dexamethasone-induced changes in the actin cytoskeleton and alters extracellular matrix protein expression in cultured human trabecular meshwork cells. Exp Eye Res 77: 181-188, 2003.

33. Yamada KM and Geiger B: Molecular interactions in cell adhesion complexes. Curr Opin Cell Biol 9: 76-85, 1997.

34. Shen L: Tight junctions on the move: Molecular mechanisms for epithelial barrier regulation. Ann N Y Acad Sci 1258: 9-18, 2012.

35. Katsuno T, Umeda K, Matsui T, Hata M, Tamura A, Itoh M, Takeuchi K, Fujimori T, Nabeshima Y, Noda T, et al: Deficiency of zonula occludens-1causes embryonic lethal phenotype associated with defected yolk sac angiogenesis and apoptosis of embryonic cells. Mol Biol Cell 19: 2465-2475, 2008.

36. Yang JG, Zhou CJ, Li XY, Sun PR, Li SP and Ren BC: Alteration of UCP2 and ZO-1 expression in trabecular meshwork of neovascular glaucoma patients. J Glaucoma 24: 291-296, 2015.

37. Yang X, Liu B, Bai Y, Chen M, Li Y, Chen M, Wei Y, Ge J and Zhuo Y: Elevated pressure downregulates ZO-1 expression and disrupts cytoskeleton and focal adhesion in human trabecular meshwork cells. Mol Vis 17: 2978-2985, 2011.

38. Zhuo YH, He Y, Leung KW, Hou F, Li YQ, Chai F and Ge J. Dexamethasone disrupts intercellular junction formation and cytoskeleton organization in human trabecular meshwork cells. Mol Vis 16: 61-71, 2010.
39. Yan Q, Clark JI and Sage EH: Expression and characterization of SPARC in human lens and in the aqueous and vitreous humors. Exp Eye Res 71: 81-90, 2000.

40. Gilbert RE, Cox AJ, Kelly DJ, Wilkinson-Berka JL, Sage EH, Jerums $G$ and Cooper ME: Localization of secreted protein acidic and rich in cysteine (SPARC) expression in the rat eye. Connect. Tissue Res 40: 295-303, 1999.

41. Rodriguez IR, Moreira EF, Bok D and Kantorow M: Osteonectin/SPARC secreted by RPE and localized to the outer plexiform layer of the monkey retina. Invest Ophthalmol Vis Sci 41: 2438-2444, 2000.

42. Tomarev SI, Wistow G, Raymond V, Dubois S and Malyukova I: Gene expression profile of the human trabecular meshwork: NEIBank sequence tag analysis. Invest Ophthalmol Vis Sci 44: 2588-2596, 2003.

43. Comes $\mathrm{N}$ and Borras $\mathrm{T}$ : Individual molecular response to elevated intraocular pressure in perfused postmortem human eyes. Physiol Genomics 38: 205-225, 2009.

44. Swaminathan SS, Oh DJ, Kang MH, Shepard AR, Pang I and Rhee DJ: TGF 32 -mediated ocular hypertension is attenuated in SPARC-null mice. Invest Ophthalmol Vis Sci 55: 4084-4097, 2014.

This work is licensed under a Creative Commons Attribution-NonCommercial-NoDerivatives 4.0 International (CC BY-NC-ND 4.0) License. 